\title{
Review Article \\ Role of Metformin on Osteoblast Differentiation in Type 2 Diabetes
}

\author{
Lin Jiating $\mathbb{D}^{1},{ }^{1}$ Ji Buyun $\left(\mathbb{D},{ }^{1}\right.$ and Zhang Yinchang $\mathbb{D}^{2}$ \\ ${ }^{1}$ Department of Stomatology, The First Affiliated Hospital of Wannan Medical College, Wuhu, Anhui Province 241000, China \\ ${ }^{2}$ Department of Orthopedics, The First Affiliated Hospital of Wannan Medical College, Wuhu, Anhui Province 241000, China
}

Correspondence should be addressed to Zhang Yinchang; 20141247@wnmc.edu.cn

Received 21 April 2019; Accepted 29 October 2019; Published 26 November 2019

Academic Editor: Lap Ho

Copyright (c) 2019 Lin Jiating et al. This is an open access article distributed under the Creative Commons Attribution License, which permits unrestricted use, distribution, and reproduction in any medium, provided the original work is properly cited.

Metformin, an effective hypoglycemic, can modulate different points of malignant mass, polycystic ovary syndrome (PCOS), cardiovascular diseases, tuberculosis, and nerve regeneration. Recently, the effect of metformin on bone metabolism has been analyzed. Metformin relies on organic cation transporters (OCT1), a polyspecific cell membrane of the solute carrier $22 \mathrm{~A}$ (SLC22A) gene family, to facilitate its intracellular uptake and action on complex I of the respiratory chain of mitochondria. These changes activate the cellular energy sensor AMP-activated protein kinase (AMPK). Thus, the increased cellular AMP/ATP ratio causes a dramatic and progressive activation of insulin and lysosomes, resulting in a decrease in intracellular glucose level, which promotes osteoblast proliferation and differentiation. AMPK also phosphorylates runt-related transcription factor 2 (Runx2) at S118, the lineage-specific transcriptional regulators, to promote osteogenesis. Metformin phosphorylates extracellular signalregulated kinase (ERK), stimulates endothelial and inducible nitric oxide synthases (e/iNOS), inhibits the GSK3 $\beta /$ Wnt $/ \beta$-catenin pathway, and promotes osteogenic differentiation of osteoblasts. The effect of metformin on hyperglycemia decreases intracellular reactive oxygen species (ROS) and advanced glycation end-products (AGEs) in collagen, and reduced serum levels of insulin-like growth factors (IGF-1) were beneficial for bone formation. Metformin has a certain effect on microangiopathy and anti-inflammation, which can induce osteoporosis, activate the activity of osteoclasts, and inhibit osteoblast activity, and has demonstrated extensive alteration in bone and mineral metabolism. The aim of this review was to elucidate the mechanisms of metformin on osteoblasts in insulin-deficient diabetes.

\section{Introduction}

Metformin, a wide spectrum of efficacy, safety hypoglycemic agents $[1,2]$, was introduced as a medication for type 2 diabetes (T2D) in 1957. As the first-line oral antihyperglycemic agent, metformin is recommended in all stages of either monotherapy or the therapy combined with other oral antihyperglycemic drugs and insulin [1,3,4]. Metformin exerts its effect on insulin-sensitive organs or tissues, such as the liver, skeletal muscle, and adipose tissues [5]. It regulates glucose homeostasis mainly by inhibiting hepatic gluconeogenesis [6]. Data have showed the beneficial effects of metformin on diabetes-associated conditions, like malignancies [7-10], inflammation [11-13], and heart failure [14]. The effect of metformin on bone metabolism in humans has been studied. A multicenter study showed metformin treatment may decrease fasting bone turnover markers representing bone resorption, suggesting that metformin may have beneficial effects on bones in diabetic patients [15]. Metformin also decreased the fracture rate in patients in vivo $[16,17]$ and promoted the osteogenesis of osteoblasts in culture [18-20]. The findings showed that metformin could prevent bone loss in ovariectomized (OVX) rats and inhibit receptor activator of nuclear factor $k$ B ligand- (RANKL-) induced osteoclast differentiation in Raw264.7 macrophage cells [21]. Moreover, a lot of data showed a long-term protective effect of metformin in bone metabolism $[22,23]$ of diabetic or prediabetic patients $[24,25]$.

Diabetes mellitus is manifested with abnormal bone mineral density (BMD), hyperglycemia, secondary calcium imbalance, disturbances in vitamin D [26], microvascular disease [27], and an increased risk of fall [28, 29]. With a 
high morbidity and mortality, it also adversely affects bone metabolism and increases the fracture risk [30-32]. Hyperglycemia also disrupts the production of ROS and AGE, which affects cell death processes [33, 34].

Several studies have reported that metformin has a potential osteogenic effect by promoting the differentiation of preosteoblasts and MSCs [19, 35, 36]. Likewise, metformin can also revert the deleterious effects of high-level glucose on osteoblastic cells [37-39]. Clinical studies have also indicated that locally delivered metformin can normalize clinical parameters of chronic periodontitis [40]. How metformin affects osteoblast differentiation is undetermined. In the present review, we explored the molecular mechanisms of metformin on osteoblasts in type 2 diabetes (Figure 1).

\section{Methods}

Using the following terms, "bone," "skeleton," "osteoblasts," "osteocytes," "osteoclasts," "bone abnormalities," "osteoporosis," "osteopenia," "idiopathic skeletal hyperostosis," "perimenopausal bone loss," "bone metabolism," "insulin sensitivity," "type 2 diabetes," "prediabetes," "metformin," and "biguanides" were searched in PubMed and the Cochrane Library for RCTs, reviews, and meta-analyses. We included relevant references in the aforementioned articles and performed hand searches for older publications.

\section{Metformin and Cell Membrane Organic Cation Transporters (OCTs)}

Metformin is a hydrophilic compound charged positively at physiological $\mathrm{pH}$. Its hydrophilicity decides its permeability through lipid membranes. The hydrophility of metformin is achieved by the polyspecific cell membrane organic cation transporters (OCTs), the solute carrier 22A (SLC22A) gene family [41-43], and multidrug and toxin extrusion (MATEs), encoded by the gene SLC47A1 [42, 44, 45]. The transporters weaken bases in an electrogenic manner to facilitate the intracellular uptake and action of metformin [46]. The present study shows that osteoblasts can transport metformin into cells through active rOCT1 (SLC22A1), and also high-level glucose can improve the uptake of metformin by osteoblasts through phosphorylating rOCT1 [47], which leads to a intracellular concentration of metformin that is stable and associated with the amount and activity of organic cation transporters, as well as metformin plasma concentration.

Since metformin carries a positive charge and can cross the plasma membrane and mitochondrial inner membrane (positive outside), metformin can enter the mitochondria [48, 49], where the concentration of metformin can rise up to 1000-fold higher than that in the extracellular circumstance to provide sufficient energy for the following biochemical reaction.

\section{Direct Effect of Metformin on Osteogenesis}

4.1. AMP-Activated Protein Kinase (AMPK) Signaling Pathway. Recently, the action of metformin in mitochondria has been intensively studied by researchers aiming to find out the osteogenic mechanism of metformin. The inhibition of complex I of the respiratory chain was highly valued $[48,50,51]$, for it was proven to suppress ATP production and increase the ratios of cytoplasmic ADP to ATP and AMP to ATP ratios (a drop in ATP level and an increase in AMP level). These changes activated AMP-activated protein kinase (AMPK) [52]. Thus, the increased cellular AMP/ATP ratio caused a progressive activation of AMPK.

Mammalian AMPK comprises $\alpha, \beta$, and $\gamma$ subunits in a heterotrimeric complex $[53,54]$. The $\alpha$ subunit has two isoforms, $\alpha 1$ and $\alpha 2$, and contains a kinase domain at the $\mathrm{N}$ terminus that is phosphorylated at Thr172 by upstream kinases [55]. The $\alpha 2$ subunit is highly expressed in skeletal and cardiac muscles and the liver [56]. The AMPK subunits displayed differential tissue-specific expression and activation [57]. AMPK $\alpha 2$ subunit was highly expressed in bone tissues, primary osteoblasts, osteoclasts, and osteoblastic cell lines [58]. AMPK also limited energy utilization to ensure cell survival, so activation of AMPK can mediate cell cycle arrest, inhibit cell growth, and suppress protein synthesis through downregulating the mechanistic target of rapamycin (MTOR, also known as the mammalian target of rapamycin) signaling [59, 60]. Metformin has been uniformly shown as a potent stimulator of AMPK activation in osteoblasts [21, 61]. The downstream target of AMPK was also phosphorylated by metformin in primary osteoblasts and the acetyl-coA carboxylase 1 (ACC1) and ACC2 isoforms of ACC phosphorylated, a process inhibiting fat synthesis, promoting fat oxidation, enhancing insulin sensitivity [62], and consequently initiating the acute inhibition of gluconeogenesis [63].

Several studies have examined the effects of AMPK activators on osteoblast differentiation, showing that metformin harbored direct osteogenic effects, such as maintaining BMD, antagonizing bone loss, and protecting bone microarchitecture $[15,18,24,64]$. AMPK signaling activation may stimulate bone formation and increase bone mass in skeletal physiology. However, the phosphorylation level of AMPK $\alpha$ subunits showed different results in the osteoblast differentiation, which was associated with decreased AMPK activity [58]. It is found that AMPK activity decreased with time during osteoblast differentiation, due to the high energy requirements and therefore elevated ATP levels in mature osteoblasts during bone matrix production and mineralization. Osteoblast differentiation coincided with the changes in cellular metabolism and mitochondrial activity [65]. Thus, metformin might activate the AMPK signal pathway in bone cells, and the mechanism linking AMPK activation with osteoblast differentiation and bone mass formation remains unknown.

4.2. Expression of Runt-Related Transcription Factor 2 (Runx2). Recently studies indicated that metformin reduced the bone loss in vivo, partly through increasing bone formation via the induction of osteoblast genes such as Runx2 and Lrp5 [66], reducing receptor activator of RANKL level, and stimulating osteoprotegerin (OPG) expression in osteoblasts [21]. 


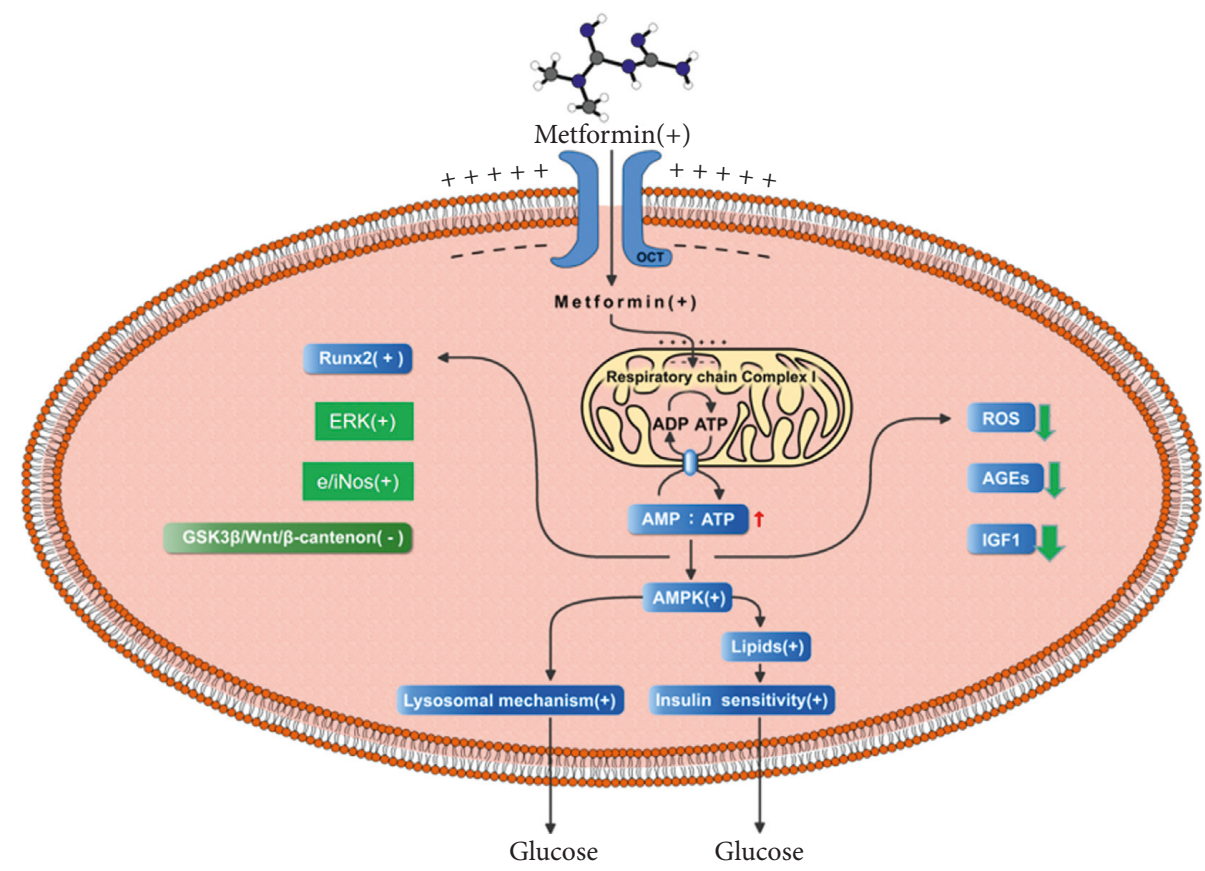

Figure 1: Potential mechanisms of metformin on osteoblasts in insulin-deficient diabetes. Metformin inhibits the respiratory chain complex I leading to an increase in AMP : ATP ratio and then stimulates 5'-AMP-activated protein kinase (AMPK), promotes glycolipid metabolism, phosphorylates runt-related transcription factor 2 (Runx2) and extracellular signal-regulated kinase (ERK), stimulates endothelial and inducible nitric oxide synthases (e/iNOS), and reduces reactive oxygen species (ROS) production, advanced glycation end-products (AGEs), and insulin-like growth factors (IGF-1).

Runt-related transcription factor 2 (Runx2), a member of the runt domain gene family, promoted the differentiation of committed mesenchymal cells or preosteoblasts into osteoblasts by regulating a range of factors, such as alkaline phosphatase (ALP), osteocalcin (OCN), and bone sialoprotein (BSP) $[67,68]$. Runx2 phosphorylation is critical in osteogenic commitment, and loss of Runx 2 may activate the adipogenic process, which shows direct relation of AMPKmediated Runx2 phosphorylation at serine 118 [69]. Metformin induced an osteogenic effect in vivo and in vitro, possibly mediated by Runx 2 and activation of AMPK $[19,38,70]$. Metformin partially inhibited the adipogenic action of BMPC and stimulated bone healing in a craniotomy defect model in both control and diabetic rats [71]. Metformin was also found to stimulate osteoblastic bone formation in MC3T3-E1 cells by regulating small heterodimer partner, an orphan nuclear receptor, that can interact with Runx2 [18]. Metformin increased ALP activity, collagen synthesis, osteocalcin production, and extracellular calcium deposition in bone marrow mesenchymal cells in vitro, possibly by upregulating the expression of Runx2 $[18,38,64]$. While controversies remain, it seems certain that some of the acute effects of metformin regulate osteoblasts by the AMPK-Runx2 signal pathway.

4.3. Other Molecular Signal Pathways. Metformin induced activation of phosphorylated extracellular signal-regulated kinase (ERK) 1/2 (p42 and p44 MAPK) and stimulated the expression of endothelial and inducible nitric oxide synthases (e/iNOS) [19]. It has been showed that increased proliferation and differentiation of osteoblast-like cells (UMR106 and MC3T3E1) was accompanied with elevated type-I collagen production and ALP activity. Metformin promoted osteogenic differentiation of hBMSCs by inhibiting the GSK3 $\beta / \mathrm{Wnt} / \beta$-catenin pathway [20]. Moreover, metformin substantially increased mineralized nodule formation of iPSC-MSC by the LKB1/AMPK pathway [18].

\section{Indirect Effect of Metformin on Osteogenesis}

Diabetes affects bones through impairing glucose metabolism, disrupting bone microvascular function and muscle endocrine function, and producing glucose oxidative derivatives [72]. Chronic hyperglycemia contributed to bone loss by modulating osteoblast gene expression, function, and bone formation. Bone metabolism was disturbed by various abnormalities, including hyperinsulinemia, deposition of advanced glycosylation end-products (AGEs) in collagen, reduced serum levels of IGF-1, hypercalciuria, renal failure, microangiopathy, and inflammation [73]. Among them, AGEs impair osteoblastic function, increasing the risk of fracture and postpone fracture in type 2 diabetes [21-25].

High-level glucose $(>44 \mathrm{mmol} / \mathrm{L})$ significantly decreased the gene expression levels of Runx2, IGF-1, and IGF-1R [38], which inhibited osteoblastic proliferation and development. Metformin increased the expression of OPG and decreased the RANKL/OPG ratio in the implant medullary area, yielding some molecular benefits in the osseointegration of implants under the hyperglycemic state, which suppressed the cell growth and mineralization [74]. Moreover, highlevel glucose increased the intracellular cAMP level in a 
time-dependent manner, which reduces bone mass and negatively regulates osteoclastogenesis [75]. These results indicate that high-level glucose can increase adipogenic and inhibit osteogenic differentiation in bone marrow stem cells.

Excessive accumulation of reactive oxygen species (ROS) - the radical forms of oxygen-contributes to agerelated changes in bones [76, 77]. ROS affects not only osteogenesis but also stimulates osteoblastic apoptosis. Excessive generation of ROS failed bone regeneration in inflammatory conditions [78, 79]. The complications of diabetes accelerated osteoblasts aging and apoptosis although the exact mechanism is not distinctly.

Hyperglycemia, hyperinsulinemia, and excessive ROS alter the microenvironment of bone cells, disturb bone microstructure, decrease bone formation, increase bone resorption, and prolong fracture healing.

\section{Conclusion and Future Perspectives}

In conclusion, metformin, an activator of AMPK, may play an important role in osteoblast differentiation and bone formation. This may be associated with the direct effect of the AMPK signaling pathway, Runx2/AMPK signaling pathway, and the indirect effect or interaction effect of metformin. Recently, our experiment has found that local injection of metformin enhanced the autophagy of aging bone marrow stromal cells (BMSCs) in vivo and accelerated new bone formation of peri-implant in vitro. The study, through the colony-forming efficiency assay, osteogenic induction and calcium accumulation, and analysis of oxidative stress, showed that metformin increased the stemness of jawbone BMSCs and accelerated new bone formation of peri-implant, both contributing to the osseointegration. Our findings highlighted the therapeutic potential of metformin in site preservation and implant in stomatology. The exact intracellular mechanism of metformin on osteoblast is ominous, and it promotes osteogenesis unambiguously.

\section{Conflicts of Interest}

The authors declare that there are no conflicts of interest.

\section{Acknowledgments}

The authors would like to thank the Department of Oral and Maxillofacial Surgery, the Affiliated Stomatology Hospital, Nanjing Medical University, Nanjing, China for supporting this project.

\section{References}

[1] C. A. Bannister, S. E. Holden, S. Jenkins-Jones et al., "Can people with type 2 diabetes live longer than those without? A comparison of mortality in people initiated with metformin or sulphonylurea monotherapy and matched, non-diabetic controls," Diabetes, Obesity and Metabolism, vol. 16, no. 11, pp. 1165-1173, 2014.

[2] R. A. Defronzo and A. M. Goodman, "Efficacy of metformin in patients with non-insulin-dependent diabetes mellitus,"
New England Journal of Medicine, vol. 333, no. 9, pp. 541-549, 1995.

[3] S. Bajaj, "RSSDI clinical practice recommendations for the management of type 2 diabetes mellitus 2017," International Journal of Diabetes in Developing Countries, vol. 38, no. S1, pp. 1-115, 2018.

[4] J. M. Campbell, S. M. Bellman, M. D. Stephenson, and K. Lisy, "Metformin reduces all-cause mortality and diseases of ageing independent of its effect on diabetes control: a systematic review and meta-analysis," Ageing Research Reviews, vol. 40, pp. 31-44, 2017.

[5] G. Rena, D. G. Hardie, and E. R. Pearson, "The mechanisms of action of metformin," Diabetologia, vol. 60, no. 9, pp. 15771585, 2017.

[6] J. J. Howell, K. Hellberg, M. Turner et al., "Metformin inhibits hepatic mTORC1 signaling via dose-dependent mechanisms involving AMPK and the TSC complex," Cell Metabolism, vol. 25, no. 2, pp. 463-471, 2017.

[7] M. Li, X. Li, H. Zhang, and Y. Lu, "Molecular mechanisms of metformin for diabetes and cancer treatment," Frontiers in Physiology, vol. 9, p. 1039, 2018.

[8] Z.-J. Zhang, Y. Bi, S. Li et al., "Reduced risk of lung cancer with metformin therapy in diabetic patients: a systematic review and meta-analysis," American Journal of Epidemiology, vol. 180, no. 1, pp. 11-14, 2014.

[9] V. Sinai Talaulikar, T. Tang, and E. Yasmin, "Role of metformin in womens health: review of its current place in clinical practice and emerging indications for future," Obstetrical \& Gynecological Survey, vol. 71, no. 5, pp. 307-317, 2016.

[10] H. J. Murff, C. L. Roumie, R. A. Greevy et al., "Metformin use and incidence cancer risk: evidence for a selective protective effect against liver cancer," Cancer Causes Control, vol. 29, no. 9, pp. 823-832, 2018.

[11] F. Malik, S. F. Mehdi, H. Ali et al., "Is metformin poised for a second career as an antimicrobial?," Diabetes/Metabolism Research and Reviews, vol. 34, no. 4, p. e2975, 2018.

[12] B. I. Restrepo, "Metformin: candidate host-directed therapy for tuberculosis in diabetes and non-diabetes patients," Tuberculosis, vol. 101, pp. S69-S72, 2016.

[13] A. R. Cameron, V. L. Morrison, D. Levin et al., "Anti-inflammatory effects of metformin irrespective of diabetes status," Circulation Research, vol. 119, no. 5, pp. 652-665, 2016.

[14] A. Dziubak, G. Wójcicka, A. Wojtak, and J. Bełtowski, "Metabolic effects of metformin in the failing heart," International Journal of Molecular Sciences, vol. 19, no. 10, p. 2869, 2018.

[15] T. B. Stage, M.-M. Hougaard Christensen, N. R. Jørgensen et al., "Effects of metformin, rosiglitazone and insulin on bone metabolism in patients with type 2 diabetes," Bone, vol. 112, pp. 35-41, 2018.

[16] P. Vestergaard, L. Rejnmark, and L. Mosekilde, "Relative fracture risk in patients with diabetes mellitus, and the impact of insulin and oral antidiabetic medication on relative fracture risk," Diabetologia, vol. 48, no. 7, pp. 1292-1299, 2005.

[17] E. Losada, B. Soldevila, M. S. Ali et al., "Real-world antidiabetic drug use and fracture risk in 12,277 patients with type 2 diabetes mellitus: a nested case-control study," Osteoporosis International, vol. 29, no. 9, pp. 2079-2086, 2018.

[18] P. Wang, T. Ma, D. Guo et al., "Metformin induces osteoblastic differentiation of human induced pluripotent stem cell-derived mesenchymal stem cells," Journal of Tissue Engineering and Regenerative Medicine, vol. 12, no. 2, pp. 437446, 2018. 
[19] A. M. Cortizo, C. Sedlinsky, A. D. McCarthy, A. Blanco, and L. Schurman, "Osteogenic actions of the anti-diabetic drug metformin on osteoblasts in culture," European Journal of Pharmacology, vol. 536, no. 1-2, pp. 38-46, 2006.

[20] J. Ma, Z. L. Zhang, X. T. Hu, X. T. Wang, and A. M. Chen, "Metformin promotes differentiation of human bone marrow derived mesenchymal stem cells into osteoblast via GSK3 $\beta$ inhibition," European Review for Medical and Pharmacological Sciences, vol. 22, no. 22, pp. 7962-7968, 2018.

[21] Q.-G. Mai, Z.-M. Zhang, S. Xu et al., "Metformin stimulates osteoprotegerin and reduces RANKL expression in osteoblasts and ovariectomized rats," Journal of Cellular Biochemistry, vol. 112, no. 10, pp. 2902-2909, 2011.

[22] W. Mu, Z. Wang, C. Ma et al., "Metformin promotes the proliferation and differentiation of murine preosteoblast by regulating the expression of sirt6 and oct4," Pharmacological Research, vol. 129, pp. 462-474, 2018.

[23] Q. Gu, Y. Gu, H. Yang, and Q. Shi, "Metformin enhances osteogenesis and suppresses adipogenesis of human chorionic villous mesenchymal stem cells," The Tohoku Journal of Experimental Medicine, vol. 241, no. 1, pp. 13-19, 2017.

[24] M. J. Tolosa, S. R. Chuguransky, C. Sedlinsky et al., "Insulindeficient diabetes-induced bone microarchitecture alterations are associated with a decrease in the osteogenic potential of bone marrow progenitor cells: preventive effects of metformin," Diabetes Research and Clinical Practice, vol. 101, no. 2, pp. 177-186, 2013.

[25] J. Sun, J. Du, W. Feng et al., "Histological evidence that metformin reverses the adverse effects of diabetes on orthodontic tooth movement in rats," Journal of Molecular Histology, vol. 48, no. 2, pp. 73-81, 2017.

[26] A. Hussain, O. B. Latiwesh, A. Ali, E. Tabrez, L. Mehra, and F. Nwachukwu, "Parathyroid gland response to vitamin D deficiency in type 2 diabetes mellitus: an observational study," Cureus, vol. 10, Article ID e3656, 2018.

[27] S. Park, H. J. Kang, J. H. Jeon, M. J. Kim, and I. K. Lee, "Recent advances in the pathogenesis of microvascular complications in diabetes," Archives of Pharmacal Research, vol. 42, no. 3, pp. 252-262, 2019.

[28] G. Li, J. C. Prior, W. D. Leslie et al., "Frailty and risk of fractures in patients with type 2 diabetes," Diabetes Care, vol. 42, no. 4, pp. 507-513, 2019.

[29] J. Starup-Linde, K. Hygum, and B. L. Langdahl, "Skeletal fragility in type 2 diabetes mellitus," Endocrinology and Metabolism, vol. 33, no. 3, pp. 339-351, 2018.

[30] N. Napoli, M. Chandran, D. D. Pierroz, B. Abrahamsen, A. V. Schwartz, and S. L. Ferrari, "Mechanisms of diabetes mellitus-induced bone fragility," Nature Reviews Endocrinology, vol. 13, no. 4, pp. 208-219, 2017.

[31] M. Janghorbani, R. M. Van Dam, W. C. Willett, and F. B. Hu, "Systematic review of type 1 and type 2 diabetes mellitus and risk of fracture," American Journal of Epidemiology, vol. 166, no. 5, pp. 495-505, 2007.

[32] A. Moayeri, M. Mohamadpour, S. Mousavi, E. Shirzadpour, S. Mohamadpour, and M. Amraei, "Fracture risk in patients with type 2 diabetes mellitus and possible risk factors: a systematic review and meta-analysis," Therapeutics and Clinical Risk Management, vol. 13, pp. 455-468, 2017.

[33] C. M. O. Volpe, P. H. Villar-Delfino, P. M. F. Dos Anjos, and J. A. Nogueira-Machado, "Cellular death, reactive oxygen species (ROS) and diabetic complications," Cell Death \& Disease, vol. 9, no. 2, p. 119, 2018.

[34] S. L. Fishman, H. Sonmez, C. Basman, V. Singh, and L. Poretsky, "The role of advanced glycation end-products in the development of coronary artery disease in patients with and without diabetes mellitus: a review," Molecular Medicine, vol. 24, no. 1, p. 59, 2018.

[35] C. Sedlinsky, M. S. Molinuevo, A. M. Cortizo et al., "Metformin prevents anti-osteogenic in vivo and ex vivo effects of rosiglitazone in rats," European Journal of Pharmacology, vol. 668, no. 3, pp. 477-485, 2011.

[36] S. Bahrambeigi, B. Yousefi, M. Rahimi, and V. Shafiei-Irannejad, "Metformin; an old antidiabetic drug with new potentials in bone disorders," Biomedicine \& Pharmacotherapy, vol. 109, pp. 1593-1601, 2019.

[37] L. Schurman, A. McCarthy, C. Sedlinsky et al., "Metformin reverts deleterious effects of advanced glycation end-products (AGEs) on osteoblastic cells," Experimental and Clinical Endocrinology \& Diabetes, vol. 116, no. 06, pp. 333-340, 2008.

[38] D. Zhen, Y. Chen, and X. Tang, "Metformin reverses the deleterious effects of high glucose on osteoblast function," Journal of Diabetes and its Complications, vol. 24, no. 5, pp. 334-344, 2010.

[39] K. Marycz, K. A. Tomaszewski, K. Kornicka et al., "Metformin decreases reactive oxygen species, enhances osteogenic properties of adipose-derived multipotent mesenchymal stem cells in vitro, and increases bone density in vivo," Oxidative Medicine and Cellular Longevity, vol. 2016, Article ID 9785890, 1 page, 2016.

[40] A. R. Pradeep, N. S. Rao, S. B. Naik, and M. Kumari, "Efficacy of varying concentrations of subgingivally delivered metformin in the treatment of chronic periodontitis: a randomized controlled clinical trial," Journal of Periodontology, vol. 84, no. 2, pp. 212-220, 2013.

[41] G. G. Graham, J. Punt, M. Arora et al., "Clinical pharmacokinetics of metformin," Clinical Pharmacokinetics, vol. 50, no. 2, pp. 81-98, 2011.

[42] H. Takane, E. Shikata, K. Otsubo, S. Higuchi, and I. Ieiri, "Polymorphism in human organic cation transporters and metformin action," Pharmacogenomics, vol. 9, no. 4, pp. 415-422, 2008.

[43] L. Chen, B. Pawlikowski, A. Schlessinger et al., "Role of organic cation transporter 3 (SLC22A3) and its missense variants in the pharmacologic action of metformin," Pharmacogenetics and Genomics, vol. 20, no. 11, pp. 687-699, 2010.

[44] T. Sato, S. Masuda, A. Yonezawa, Y. Tanihara, T. Katsura, and K.-I. Inui, "Transcellular transport of organic cations in double-transfected MDCK cells expressing human organic cation transporters hOCT1/hMATE1 and hOCT2/hMATE1," Biochemical Pharmacology, vol. 76, no. 7, pp. 894-903, 2008.

[45] M. Tsuda, T. Terada, M. Ueba et al., "Involvement of human multidrug and toxin extrusion 1 in the drug interaction between cimetidine and metformin in renal epithelial cells," Journal of Pharmacology and Experimental Therapeutics, vol. 329, no. 1, pp. 185-191, 2009.

[46] A. T. Nies, H. Koepsell, K. Damme, and M. Schwab, “Organic cation transporters (OCTs, MATEs), in vitro and in vivo evidence for the importance in drug therapy," Handbook of Experimental Pharmacology, vol. 201, pp. 105-167, 2011.

[47] L. Ma, X. Wu, E. Ling-Ling, D.-S. Wang, and H.-C. Liu, “The transmembrane transport of metformin by osteoblasts from rat mandible," Archives of Oral Biology, vol. 54, no. 10, pp. 951-962, 2009.

[48] M. R. Owen, E. Doran, and A. P. Halestrap, "Evidence that metformin exerts its anti-diabetic effects through inhibition of complex 1 of the mitochondrial respiratory chain," Biochemical Journal, vol. 348, no. 3, pp. 607-614, 2000. 
[49] H. R. Bridges, A. J. Y. Jones, M. N. Pollak, and J. Hirst, "Effects of metformin and other biguanides on oxidative phosphorylation in mitochondria," Biochemical Journal, vol. 462, no. 3 , pp. 475-487, 2014.

[50] M.-Y. El-Mir, V. Nogueira, E. Fontaine, N. Avéret, M. Rigoulet, and X. Leverve, "Dimethylbiguanide inhibits cell respiration via an indirect effect targeted on the respiratory chain complex I," Journal of Biological Chemistry, vol. 275, no. 1, pp. 223-228, 2000.

[51] M. Foretz, B. Guigas, L. Bertrand, M. Pollak, and B. Viollet, "Metformin: from mechanisms of action to therapies," Cell Metabolism, vol. 20, no. 6, pp. 953-966, 2014.

[52] S. A. Hawley, F. A. Ross, C. Chevtzoff et al., "Use of cells expressing $\gamma$ subunit variants to identify diverse mechanisms of AMPK activation," Cell Metabolism, vol. 11, no. 6, pp. 554-565, 2010.

[53] G. Gao, C. S. Fernandez, D. Stapleton et al., "Non-catalytic - $\beta$ and $\gamma$-subunit isoforms of the 5-AMP-activated protein kinase," Journal of Biological Chemistry, vol. 271, no. 15, pp. 8675-8681, 1996.

[54] A. Woods, P. C. F. Cheung, F. C. Smith et al., "Characterization of AMP-activated protein kinase $\beta$ and $\gamma$ subunits," Journal of Biological Chemistry, vol. 271, no. 17, pp. 1028210290, 1996.

[55] K. A. David Carling, K. Aguan, A. Woods et al., "Mammalian AMP-activated protein kinase is homologous to yeast and plant protein kinases involved in the regulation of carbon metabolism," The Journal of Biological Chemistry, vol. 269, no. 15 , pp. 11442-11448, 1994.

[56] D. Stapleton, K. I. Mitchelhill, G. Gao et al., "Mammalian AMP-activated protein kinase subfamily," Journal of Biological Chemistry, vol. 271, no. 2, pp. 611-614, 1996.

[57] D. Carling, "AMPK signalling in health and disease," Current Opinion in Cell Biology, vol. 45, pp. 31-37, 2017.

[58] T. Kasai, K. Bandow, H. Suzuki et al., "Osteoblast differentiation is functionally associated with decreased AMP kinase activity," Journal of Cellular Physiology, vol. 221, no. 3, pp. 740-749, 2009.

[59] I. Tamargo-Gomez and G. Marino, "AMPK: regulation of metabolic dynamics in the context of autophagy," International Journal of Molecular Sciences, vol. 19, no. 12, p. 3812, 2018.

[60] H. Lin, Y. Ying, Y.-Y. Wang et al., "AMPK downregulates ALK2 via increasing the interaction between Smurf1 and Smad6, leading to inhibition of osteogenic differentiation," Biochimica et Biophysica Acta (BBA) - Molecular Cell Research, vol. 1864, no. 12, pp. 2369-2377, 2017.

[61] C.-S. Zhang, M. Li, T. Ma et al., "Metformin activates AMPK through the lysosomal pathway," Cell Metabolism, vol. 24, no. 4, pp. 521-522, 2016.

[62] M. D. Fullerton, S. Galic, K. Marcinko et al., "Single phosphorylation sites in Acc1 and Acc2 regulate lipid homeostasis and the insulin-sensitizing effects of metformin," Nature Medicine, vol. 19, no. 12, pp. 1649-1654, 2013.

[63] M. F. Vincent, P. J. Marangos, H. E. Gruber, and G. van den Berghe, "Inhibition by AICA riboside of gluconeogenesis in isolated rat hepatocytes," Diabetes, vol. 40, no. 10, pp. 1259-1266, 1991.

[64] C. Wang, H. Li, S.-G. Chen et al., "The skeletal effects of thiazolidinedione and metformin on insulin-resistant mice," Journal of Bone and Mineral Metabolism, vol. 30, no. 6, pp. 630-637, 2012.

[65] S. V. Komarova, F. I. Ataullakhanov, and R. K. Globus, "Bioenergetics and mitochondrial transmembrane potential during differentiation of cultured osteoblasts," American Journal of Physiology-Cell Physiology, vol. 279, no. 4, pp. C1220-C1229, 2000.

[66] Y. Gao, Y. Li, J. Xue, Y. Jia, and J. Hu, "Effect of the antidiabetic drug metformin on bone mass in ovariectomized rats," European Journal of Pharmacology, vol. 635, no. 1-3, pp. 231-236, 2010.

[67] Y. Ito, "Runx genes in development and cancer: regulation of viral gene expression and the discovery of Runx family genes," Advances in Cancer Research, vol. 99, pp. 33-76, 2008.

[68] T. Komori, "Regulation of skeletal development by the Runx family of transcription factors," Journal of Cellular Biochemistry, vol. 95, no. 3, pp. 445-453, 2005.

[69] S. Chava, S. Chennakesavulu, B. Meher Gayatri, and A. B. M. Reddy, "A novel phosphorylation by AMP-activated kinase regulates Runx2 from ubiquitination in osteogenesis over adipogenesis," Cell Death \& Disease, vol. 9, no. 7, p. 754, 2018.

[70] Y.-S. Lee, Y.-S. Kim, S.-Y. Lee et al., “AMP kinase acts as a negative regulator of RANKL in the differentiation of osteoclasts," Bone, vol. 47, no. 5, pp. 926-937, 2010.

[71] M. S. Molinuevo, L. Schurman, A. D. McCarthy et al., "Effect of metformin on bone marrow progenitor cell differentiation: in vivo and in vitro studies," Journal of Bone and Mineral Research, vol. 25, no. 2, pp. 211-221, 2010.

[72] B. Lecka-Czernik, "Diabetes, bone and glucose-lowering agents: basic biology," Diabetologia, vol. 60, no. 7, pp. 1163$1169,2017$.

[73] A. Montagnani, S. Gonnelli, M. Alessandri, and R. Nuti, "Osteoporosis and risk of fracture in patients with diabetes: an update," Aging Clinical and Experimental Research, vol. 23, no. 2, pp. 84-90, 2011.

[74] C. Serrão, M. Bastos, D. Cruz, F. Malta, P. Vallim, and P. Duarte, "Role of metformin in reversing the negative impact of hyperglycemia on bone healing around implants inserted in type 2 diabetic rats," The International Journal of Oral \& Maxillofacial Implants, vol. 32, no. 3, pp. 547-554, 2017.

[75] H. Kang, B. Viollet, and D. Wu, "Genetic deletion of catalytic subunits of AMP-activated protein kinase increases osteoclasts and reduces bone mass in young adult mice," Journal of Biological Chemistry, vol. 288, no. 17, pp. 12187-12196, 2013.

[76] T. Finkel and N. J. Holbrook, "Oxidants, oxidative stress and the biology of ageing," Nature, vol. 408, no. 6809, pp. 239-247, 2000.

[77] R. S. Balaban, S. Nemoto, and T. Finkel, "Mitochondria, oxidants, and aging," Cell, vol. 120, no. 4, pp. 483-495, 2005.

[78] S. Di Meo, T. T. Reed, P. Venditti, and V. Manuel Victor, "Role of ROS and RNS sources in physiological and pathological conditions," Oxidative Medicine and Cellular Longevity, vol. 2016, Article ID 1245049, 44 pages, 2016.

[79] C. Liu, L. Mo, Y. Niu, X. Li, X. Zhou, and X. Xu, "The role of reactive oxygen species and autophagy in periodontitis and their potential linkage," Frontiers in Physiology, vol. 8, p. 439, 2017. 


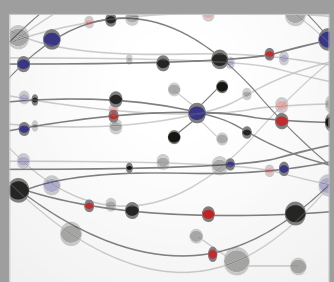

The Scientific World Journal
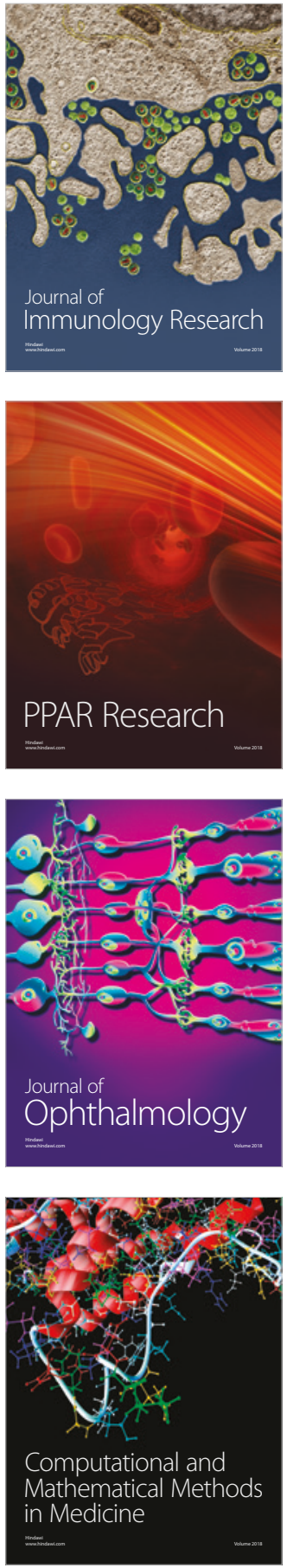

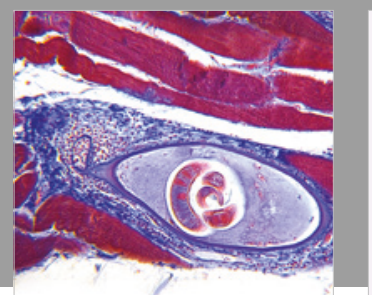

Gastroenterology Research and Practice

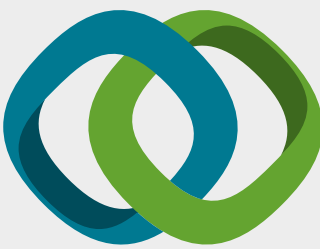

\section{Hindawi}

Submit your manuscripts at

www.hindawi.com
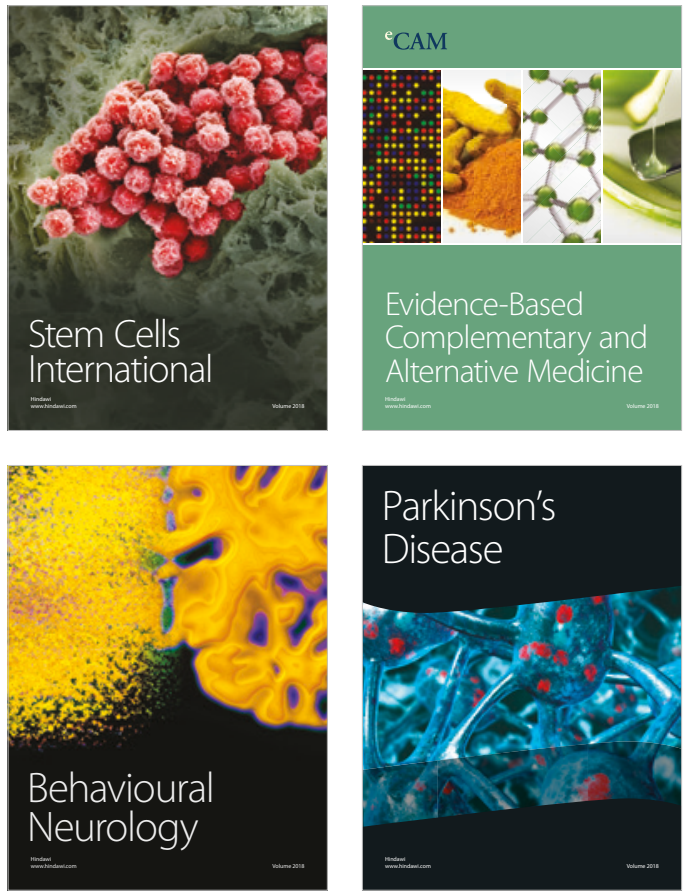

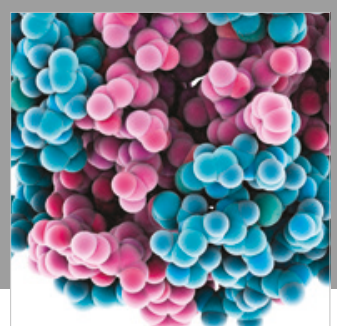

ournal of

Diabetes Research

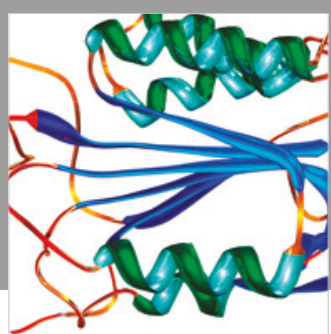

Disease Markers
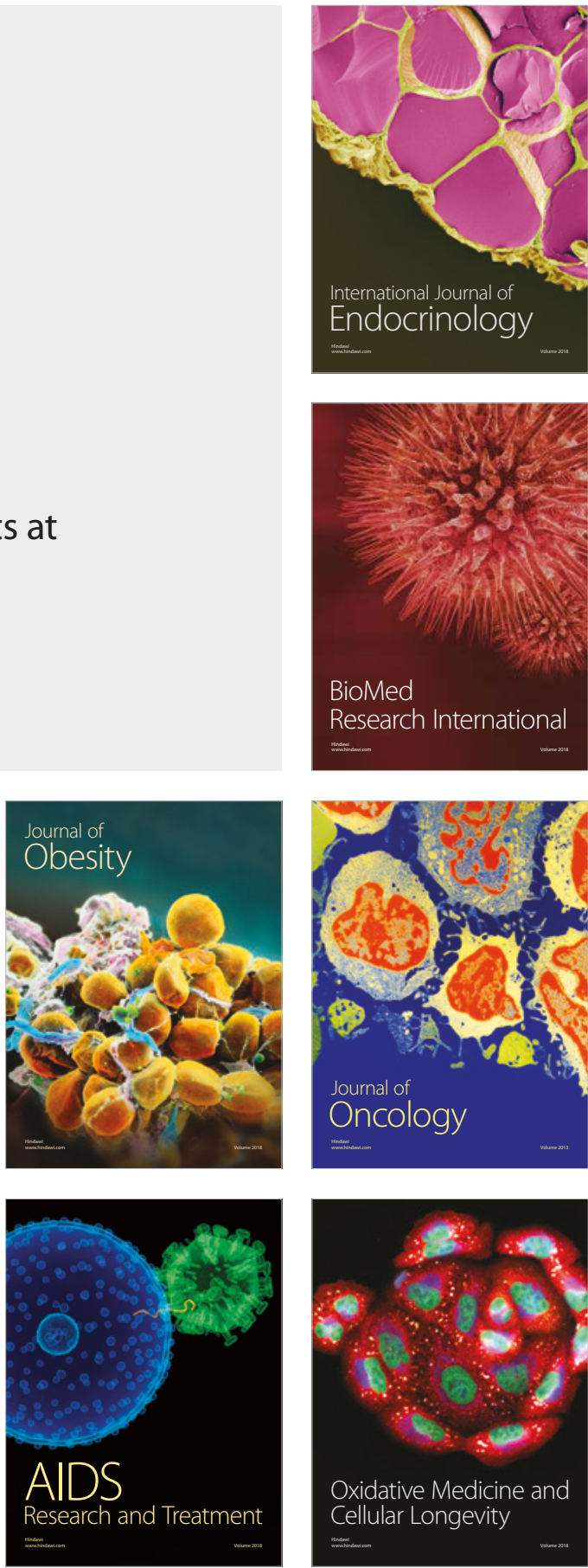